\title{
Review: in non-referred samples of children, early externalising behaviour does not predict later antisocial behaviour
}

\author{
Bennett KJ, Lipman EL, Racine Y, et al. Annotation: Do measures of externalising behaviour in normal populations predict later \\ outcome? Implications for targeted interventions to prevent conduct disorder.J Child Psychol Psychiatry 1998 Nov;39:1059-70.
}

\section{Question}

Is externalising behaviour in normal kindergarten and first grade children associated with subsequent conduct disorder and antisocial behaviour?

\section{Data sources}

\{Studies were identified by doing a systematic search of Medline, PsycINFO, and ERIC; by reviewing the bibliographies of relevant published reports; by searching personal files; and by contacting colleagues and experts in the field. * $^{*}$

\section{Study selection}

\{Studies were selected if they included a representative non-referred sample of children (inception cohorts of clinical samples were not included) and if at least 1 assessment of externalising behaviour was made in the kindergarten to grade 1 range with at least 1 subsequent follow up to assess the occurrence of conduct disorder or antisocial behaviour. * $^{*}$

\section{Data extraction}

Data were extracted on sample characteristics, assessment methods, statistical analysis, outcome measures, and study quality.

\section{Main results}

13 studies with 1 to 7 years follow up met the selection criteria. 7 of the 13 studies reported that a statistical association existed between early externalising behaviour and later development of antisocial behaviour or conduct disorder. Across the 13 studies, sensitivity (the proportion of individuals with later antisocial behaviour or conduct disorder who had externalising behaviour earlier) ranged from $28 \%$ to $100 \%$ (15 estimates) with almost two thirds of the estimates $\leqslant 50 \%$. The estimates of sensitivity $>60 \%$ were associated with low levels of specificity (the proportion of individuals without later antisocial behaviour or conduct disorder who did not have externalising behaviour earlier). Specificity ranged from $58 \%$ to $97 \%$ (14 estimates) and was > $>90 \%$ in only 2 cases. Based on a sensitivity of $45 \%$, a specificity of $85 \%$ and a prevalence of $10 \%$ (realistic estimates consistent with a nonreferred population of children), only $25 \%$ of children with externalising behaviour will have antisocial behaviour or conduct disorder (the positive predictive value, \{relative risk in a longitudinal study is $3.0,95 \%$ CI 1.2 to 6.3$\} \dagger$ ).

\section{Conclusion}

The value of using early externalising symptoms in non-referred populations of kindergarten and first grade children to predict later antisocial behaviour or conduct disorder is modest and the level of misclassification is likely to be high.

*Information provided by the author.

†Numbers calculated from data in article.

Sources of funding: MacArthur Foundation; Ontario Mental Health Foundation; Health Canada.

For correspondence: $\operatorname{Dr} K$ J Bennett, Associate Professor, Department of Clinical Epidemiology and Biostatistics, Room 3V43D, McMaster University, 1200 Main Street West, Hamilton, ON L8N 3Z5, Canada. Fax +1905 5283154 .

\section{Commentary}

Of substantial interest to clinicians, researchers, and policy makers alike is the extent to which we can effectively identify and intervene with young children at risk of conduct and behavioural problems to prevent longer term adverse consequences that are more difficult and costly to treat. Implementation of such a strategy, however, rests not just on having an effective, palatable, and affordable intervention ready to be deployed (a major feat all in itself), but also on having a reliable and valid means of identifying children truly in need of such interventions. Although previous reports may have over estimated our ability to identify such children, Bennett et al summarise information only from studies of population based samples where follow up assessments were conducted over time. Such studies provide unbiased estimates of the ability to identify children at risk of long term externalising behavioural problems.

The findings of this review are at once both heartening and bleak: based on the review, fewer than one half of children identified with early externalising problems continued to show these problems at later ages - a finding at some variance with the common notion that early aggressive problems portray a picture of uniformly grim long term consequences. On the other hand, this same difficulty in accurately predicting later outcomes poses greater burdens and requirements on potential interventions-they must be sufficiently efficacious and/or cost effective to make up for the potential problems of inadvertently labelling and/or misapplying scarce resources to those not in need. As a strategy that might be used to address these problems, Bennett et al note that 2 or 3 stage screening methods might offer an efficient means of identifying such chil- dren. In addition, they propose a unique solution, one more akin to the types of strategies used by thoughtful cliniciansnamely, children in need might be best identified not only through the presence of early behavioural signs and symptoms but also through the convergence of other indicators, such as well established risk factors for adverse outcomes independent from the behavioural indicators themselves (eg, single parent status, family poverty, neuropsychological functioning, etc). Although both strategies appear to be sensible, additional research will be required to determine whether in fact they can be deployed and used to advantage on behalf of young children in need.

Peter $\mathrm{S}$ Jensen, $\mathrm{MD}$ National Institute of Mental Health Bethesda, Maryland, USA 